\title{
Double-Expressor Phenotype (BCL-2/c-MYC Co- expression) of Diffuse Large B-Cell Lymphoma and Its Clinicopathological Correlation
}

Atif A. Hashmi ${ }^{1}$, Syeda N. Iftikhar ${ }^{1}$, Gul Nargus ${ }^{2}$, Omer Ahmed ${ }^{3}$, Ishaq Azeem Asghar ${ }^{4}$, Umme Aiman Shirazi ${ }^{1}$, Anoshia Afzal ${ }^{5}$, Muhammad Irfan ${ }^{6}$, Javaria Ali ${ }^{1}$

1. Pathology, Liaquat National Hospital and Medical College, Karachi, PAK 2. Pathology, Khyber Medical College, Peshawar, PAK 3. Internal Medicine, Liaquat National Hospital and Medical College, Karachi, PAK 4. Pathology, Ascension St. John Hospital, Detroit, USA 5. Pathology, University of Oklahoma Health Sciences Center, Oklahoma City, USA 6. Statistics, Liaquat National Hospital and Medical College, Karachi, PAK

Corresponding author: Atif A. Hashmi, atifhashmi345@gmail.com

\section{Abstract}

\section{Introduction}

Diffuse large B-cell lymphoma (DLBCL) is a heterogeneous disease, the spectrum of which is increasing with time. The 2016 World Health Organization (WHO) update on hematopoietic tumors recognized a prognostic subgroup of DLBCL called double-expressor DLBCL. Double-expressor DLBCL is defined by the coexpression of c-MYC and BCL-2 by using immunohistochemical (IHC) studies. To our knowledge, very few studies have looked into the pathological features of this newly defined prognostic category of DLBCL; therefore, in this study we evaluated the frequency of the double-expressor phenotype of DLBCL and its association with other clinicopathological parameters.

\section{Methods}

We conducted a retrospective observational study in the Department of Histopathology, Liaquat National Hospital and Medical College, from November 2017 till December 2020. Pathological and clinical records were retrieved from departmental archives. All cases diagnosed as DLBCL were included in the study. More than $40 \%$ c-MYC expression in the presence of more than 50\% BCL-2 expression was defined as doubleexpressor DLBCL.

\section{Results}

The mean age of the patients was $52.1 \pm 16.9$ years. The mean Ki67 index was $73.0 \pm 17.0 \%$. A total of $48.6 \%$ cases were of germinal center B-cell-like (GCB) subtype, and 59.6\% cases were nodal. Double-expressor phenotype was noted in $35.8 \%$ of DLBCL cases. A significant association of double-expressor phenotype was noted with age, gender, Ki67 index and subtype of DLBCL. Double-expressor DLBCL had a higher mean age than non-double-expressor DLBCL. Similarly, double-expressor DLBCL had a higher Ki67 index. Moreover,

Review began 02/01/2021 Review ended 02/01/2021 Published 02/05/2021

\section{(c) Copyright 2021}

Hashmi et al. This is an open access article distributed under the terms of the Creative Commons Attribution License CC-BY 4.0., which permits unrestricted use, distribution, and reproduction in any medium, provided the original author and source are credited. double-expressor phenotype was associated with non-GCB subtype DLBCL.

\section{Conclusion}

We found a high proportion of double-expressor phenotype DLBCL in our population. Moreover, doubleexpressor phenotype DLBCL was associated with female gender, higher age, higher Ki67 and non-GCB subtype. The association of double-expressor DLBCL with a high Ki67 index and non-GCB subtype confers a poor prognostic significance of this variant of DLBCL, requiring more aggressive therapy.

Categories: Pathology, Oncology, Hematology

Keywords: diffuse large b-cell lymphoma, germinal center subtype, non-germinal center subtype, double-expressor, c-myc, bcl-2

\section{Introduction}

Diffuse large B-cell lymphoma (DLBCL) is a heterogeneous disease, the spectrum of which is increasing with time [1]. Gene expression profiling studies have distinguished two subtypes of DLBCL, namely, germinal center B-cell-like (GCB) and activated B-cell-like (ABC) subtypes. While molecular studies are considered the gold standard for this subtyping, immunohistochemical (IHC) studies are considered surrogate for the subtyping of DLBCL into GCB and non-GCB types. Studies have shown the prognostic significance of this subtyping, with the GCB subtype being prognostically better than the non-GCB subtype [2]. The 2016 World Health Organization (WHO) update on hematopoietic tumors recognized another prognostic subgroup of DLBCL called double-expressor DLBCL. Double-expressor DLBCL is defined by the co-expression of c-MYC and BCL-2 by using IHC studies. Double-expressor DLBCL is prognostically poorer than the non-double expressor phenotype DLBCL; however, it is better than double-hit and triple-hit B-cell lymphomas, 


\section{Cureus}

prognostically. Double-hit and triple-hit B-cell lymphomas are high-grade B-cell lymphomas defined by the genetic rearrangements of c-MYC and BCL-2 and/or BCL-6, accordingly, by targeted fluorescence in situ hybridization (FISH) studies. Double-hit and triple-hit lymphomas are currently not considered subtypes of DLBCL as they prognostically behave differently than DLBCL. DLBCL is the most common non-Hodgkin's lymphoma in Pakistan [3]. To our knowledge, very few studies have looked into the pathological features of this newly defined prognostic category of DLBCL; therefore, in this study we evaluated the frequency of the double-expressor phenotype of DLBCL and its association with other clinicopathological parameters.

\section{Materials And Methods}

We conducted a retrospective observational study in the Department of Histopathology, Liaquat National Hospital and Medical College, from November 2017 till December 2020. Pathological and clinical records were retrieved from departmental archives. All cases diagnosed as DLBCL were included in the study. A panel of IHC stains was applied to diagnose DLBCL, including CD20, PAX5, CD3, CD5, SOX11, cyclin D1, CD10, and CD23. If differential diagnosis included carcinoma or melanoma, then S100 and pan-cytokeratin IHC stains were included in the initial IHC panel. Further IHC studies were performed to subcategorize DLBCL. The Hans algorithm was used to subcategorize DLBCL into GCB and non-GCB subtypes. GCB subtype was defined as more than $30 \%$ expression of CD10, or more than 30\% expression of BCL- 6 in the absence of MUM1 and CD10 expression. All other immunophenotypic expressions (CD10-/MUM1+/BCL-6+, CD10/MUM1+/BCL-6-, CD10-/MUM1-/BCL-6-) were labeled as non-GCB DLBCL. More than 40\% c-MYC expression in the presence of more than 50\% BCL-2 expression was defined as double-expressor DLBCL (Figure 1).

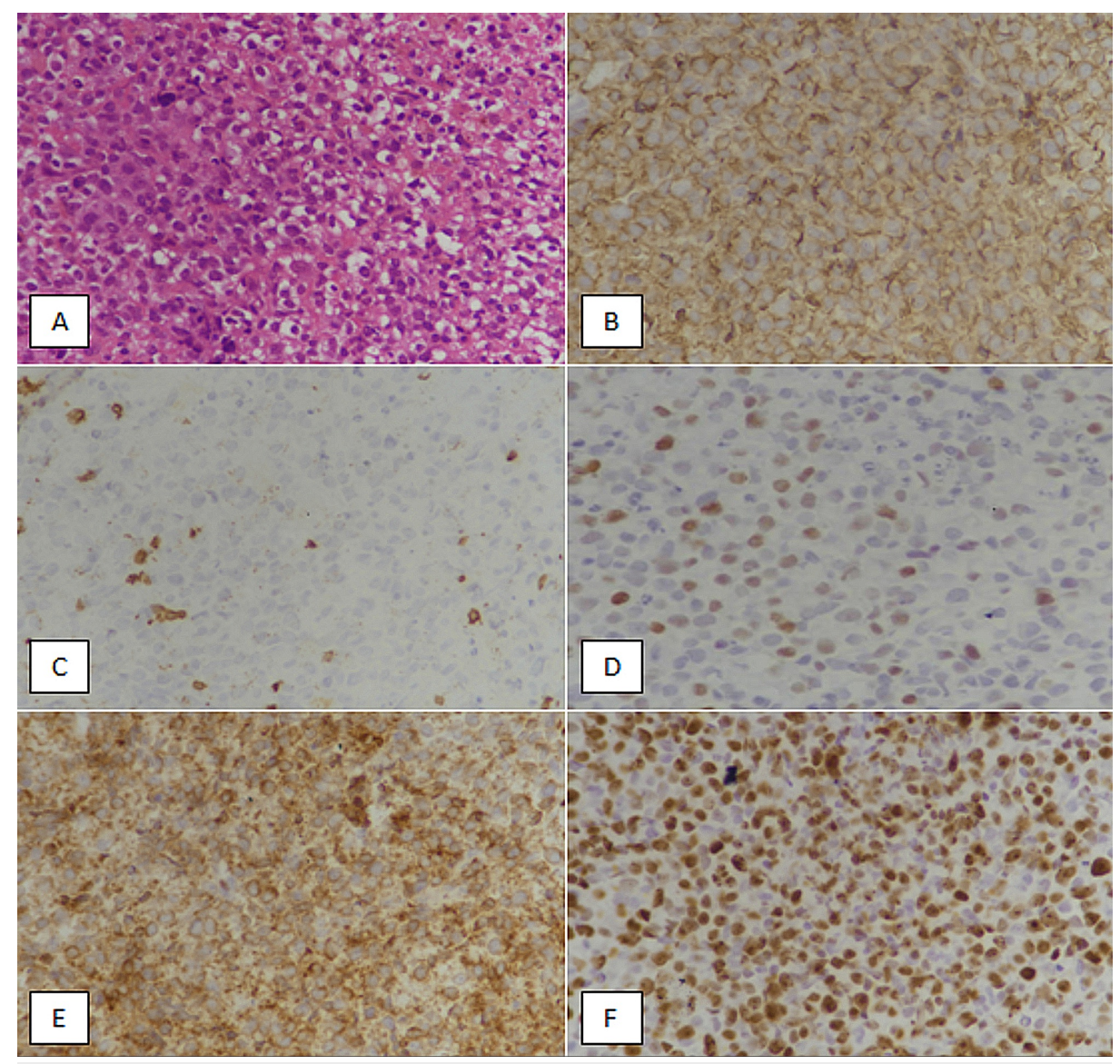

\section{FIGURE 1: Double-expressor diffuse large B-cell lymphoma}

(A) Hematoxylin and eosin-stained section at 400x magnification shows sheets of large atypical cells. (B) CD20 immunohistochemical staining shows diffuse membranous expression in atypical lymphoid cells. (C) CD3 immunostaining reveals no expression in tumor cells. Occasional background reactive T-cells are highlighted. (D) c-MYC immunohistochemical staining is depicting positive nuclear expression in more than $40 \%$ of the tumor cells. (E) BCL-2 immunohistochemical staining shows diffuse expression in lymphoma cells. (F) The Ki67 immunomarker is revealing an $85 \%$ proliferative index. 


\section{Cureus}

\section{Results}

A total of 109 DLBCL cases were included in the study. The mean age of the patients was $52.1 \pm 16.9$ years The mean Ki67 index was $73.0 \pm 17.0 \%$. A total of $48.6 \%$ cases were of GCB subtype, and $59.6 \%$ cases were nodal. Double-expressor phenotype was noted in $35.8 \%$ of DLBCL cases (Table 1).

Clinicopathological characteristics

Age (years)*

$\operatorname{Ki67}(\%)^{\star}$

Age groups

$\leq 35$ years

36-50 years

$>50$ years

Gender

Male

Female

Subtype of DLBCL

Germinal center B-cell-like subtype

53 (48.6)

Non-germinal center B-cell-like subtype

Site

Nodal

Extra-nodal

65 (59.6)

44 (40.4)

Specimen type

Trucut biopsy

Excision biopsy

BCL-2

Positive

66 (60.6)

Negative

BCL-6

Positive

51 (46.8)

Negative

58 (53.2)

MUM1

Positive

46 (42.2)

Negative

63 (57.8)

c-MYC

Positive

Negative

48 (44)

61 (56)

CD10

Positive

44 (40.4)

Negative

65 (59.6)

CD30

Positive

$2.1 \pm 16.9$

$3.0 \pm 17.0$

1 (19.3)

5 (59.6)

9 (54.1)

0 (45.9)

49 (45)

60 (55)

43 (39.4)

.2)

requency (\%)

8)




\section{Cureus}

Negative

$100(91.7)$

Double-expressor phenotype

Yes

No
39 (35.8)

70 (64.2)

\section{TABLE 1: Descriptive statistics of the study population}

DLBCL, diffuse large B-cell lymphoma

*Mean \pm standard deviation

A significant association of double-expressor phenotype was noted with age, gender, Ki67 index, and subtype of DLBCL. Double-expressor DLBCL had a higher mean age than non-double-expressor DLBCL. Similarly, double-expressor DLBCL had a higher Ki67 index. Moreover, double-expressor phenotype was associated with non-GCB-type DLBCL. No significant association was noted with respect to the site of involvement (nodal vs. extra-nodal) and CD30 IHC expression (Table 2). 


\section{Cureus}

\begin{tabular}{|c|c|c|c|}
\hline \multirow{3}{*}{ Clinicopathological characteristics } & \multicolumn{2}{|c|}{ Frequency (\%) } & \multirow{3}{*}{ p-value } \\
\hline & \multicolumn{2}{|c|}{ Double-expressor phenotype DLBCL } & \\
\hline & Yes & No & \\
\hline Age (years) ${ }^{*}$ & $59.4 \pm 12.6$ & $48.0 \pm 17.8$ & $<0.0001^{\star \star \star \star}$ \\
\hline Ki67 (\%)* & $78.1 \pm 15.2$ & $70.2 \pm 17.4$ & $0.019^{\star \star \star \star}$ \\
\hline \multicolumn{4}{|l|}{ Age groups $^{\star *}$} \\
\hline$\leq 35$ years & $3(7.7)$ & $18(25.7)$ & \multirow{3}{*}{$<0.0001^{\star \star \star \star}$} \\
\hline $36-50$ years & $3(7.7)$ & $20(28.6)$ & \\
\hline$>50$ years & $33(84.6)$ & $32(45.7)$ & \\
\hline \multicolumn{4}{|l|}{ Gender** } \\
\hline Male & 13 (33.3) & $46(65.7)$ & \multirow{2}{*}{$0.001^{\star \star \star \star}$} \\
\hline Female & $26(66.7)$ & 24 (34.3) & \\
\hline \multicolumn{4}{|l|}{ Subtype of DLBCL ${ }^{* *}$} \\
\hline Germinal center B-cell-like subtype & $14(35.9)$ & $39(55.7)$ & \multirow{2}{*}{$0.047^{\star \star \star \star}$} \\
\hline Non-germinal center B-cell-like subtype & $25(64.1)$ & $31(44.3)$ & \\
\hline \multicolumn{4}{|l|}{ Site $^{\star \star}$} \\
\hline Nodal & $25(64.1)$ & $40(57.1)$ & \multirow{2}{*}{0.478} \\
\hline Extra-nodal & $14(35.9)$ & $30(42.9)$ & \\
\hline \multicolumn{4}{|l|}{ BCL- $6^{\star \star}$} \\
\hline Positive & $21(53.8)$ & $30(42.9)$ & \multirow{2}{*}{0.270} \\
\hline Negative & $18(46.2)$ & $40(57.1)$ & \\
\hline \multicolumn{4}{|l|}{ MUM1** } \\
\hline Positive & $26(66.7)$ & $20(28.6)$ & \multirow{2}{*}{$<0.0001^{* \star \star *}$} \\
\hline Negative & $13(33.3)$ & $50(71.4)$ & \\
\hline \multicolumn{4}{|l|}{ CD10** } \\
\hline Positive & $10(25.6)$ & 34 (48.6) & \multirow{2}{*}{$0.019^{\star \star \star *}$} \\
\hline Negative & $29(74.4)$ & $36(51.4)$ & \\
\hline \multicolumn{4}{|l|}{$\mathrm{CD} 30^{\star \star \star}$} \\
\hline Positive & $3(7.7)$ & $6(8.6)$ & \multirow{2}{*}{1.000} \\
\hline Negative & $36(92.3)$ & $64(91.4)$ & \\
\hline
\end{tabular}

\section{TABLE 2: Association of double-expressor phenotype DLBCL with clinicopathological features} DLBCL, diffuse large B-cell lymphoma

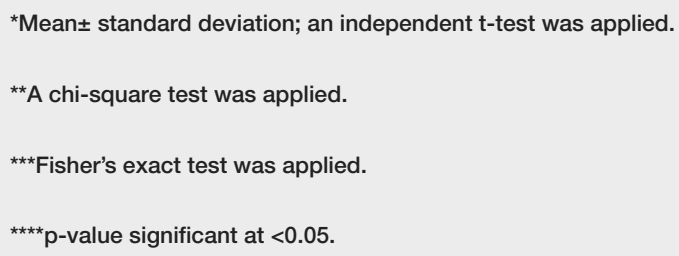




\section{Discussion}

In this study, we found that a significant proportion of DLBCL had co-expression of BCL-2 and c-MYC conferring a double-expressor phenotype according to the WHO classification. Moreover, double-expressor phenotype DLBCL was associated with female gender, higher age, higher Ki67 index and non-GCB subtype.

Some studies have proposed that the prognosis of c-MYC/BCL-2 double-expressor phenotype is worse than other subtypes of DLBCL $[4,5]$, but other studies had mixed results $[6,7]$. Nagib et al. studied doubleexpressor DLBCL and found that it was associated with an overall decreased disease-free survival [8]. Naseem et al. studied the frequency and prognosis of double-expressor DLBCL and found that the frequency of c-MYC/BCL-2 co-expression was $14 \%$, with a median survival of 10 months [9]. Conversely, we found a higher frequency of double-expressor phenotype in our study (35.8\%).

It is recommended to differentiate double-expressor DLBCL from other DLBCL subtypes as they have poor clinical behavior and need more aggressive interventions $[10,11]$. Some recent studies have confirmed that a high Ki67 proliferation index and c-MYC/BCL-2 co-expression in DLBCL were independently associated with poor clinical outcomes $[2,12,13]$. Factors such as age, sex, and proliferation index and their relationship with DLBCL subtypes should be studied in detail to fully understand the importance of each factor separately, as it might have significant clinical implications.

Owing to the expression of cMYC, the differential diagnosis of double-expressor DLBCL also includes Burkitt's lymphoma (BL). However, BL is characterized by intermediate-sized lymphoid cells with almost $100 \%$ Ki67 index, positive expression with CD10 and BCL-6, and lack of expression of BCL-2, although the same immuophenotype can be encountered in DLBCL and differentiation is sometimes difficult to make in the absence of molecular/genetic analysis.

Standard therapy for DLBCL is rituximab, cyclophosphamide, hydroxydaunorubicin (doxorubicin), Oncovin (vincristine), and prednisone (R-CHOP). Owing to the aggressive nature of double-expressor and double-hit lymphoma, more aggressive regimens than R-CHOP like EPOCH (etoposide, vincristine, doxorubicin, with cyclophosphamide and prednisone) were suggested; however, the prognosis remains dismal. Alternatively, novel-targeted agents, directly or indirectly inhibiting BCL-2 and cMYC, are being investigated and results are encouraging [14].

Our study was limited owing to the small sample size and retrospective study design. Moreover, clinical follow-up data were not available to compare survival between double-expressor and non-double expressor phenotypes of DLBCL in our study. In addition, molecular studies were not performed to evaluate genetic rearrangements of c-MYC and BCL-2.

\section{Conclusions}

In this study, we evaluated the co-expression of BCL-2 and c-MYC in DLBCL designated as the doubleexpressor phenotype DLBCL. We noted that a significant proportion of DLBCL in our study had doubleexpressor phenotype. We also found that double-expressor DLBCL had a higher Ki67 index than the nondouble-expressor DLBCL. An association of double-expressor phenotype was also noted with non-GCB-type DLBCL. As previous studies have confirmed a poor prognostic significance of a high Ki67 index and non-GCB type in DLBCL, an association of double-expressor DLBCL with non-GCB phenotype and a high Ki67 index portends a poor prognostic significance of double-expressor DLBCL. Future studies are warranted to evaluate the difference in disease-free survival of double-expressor DLBCL and other subtypes of DLBCL.

\section{Additional Information}

\section{Disclosures}

Human subjects: Consent was obtained or waived by all participants in this study. Animal subjects: All authors have confirmed that this study did not involve animal subjects or tissue. Conflicts of interest: In compliance with the ICMJE uniform disclosure form, all authors declare the following: Payment/services info: All authors have declared that no financial support was received from any organization for the submitted work. Financial relationships: All authors have declared that they have no financial relationships at present or within the previous three years with any organizations that might have an interest in the submitted work. Other relationships: All authors have declared that there are no other relationships or activities that could appear to have influenced the submitted work.

\section{References}

1. Li S, Young KH, Medeiros LJ: Diffuse large B-cell lymphoma. Pathology. 2018, 50:74-87. 10.1016/.jpathol.2017.09.006

2. Liu Y, Barta SK: Diffuse large B-cell lymphoma: 2019 update on diagnosis, risk stratification, and treatment . Am J Hematol. 2019, 94:604-616. 10.1002/aih.25460

3. Hashmi AA, Hussain ZF, Faridi N, Khurshid A: Distribution of Ki67 proliferative indices among WHO subtypes of non-Hodgkin's lymphoma: association with other clinical parameters. Asian Pac J Cancer Prev. 
2014, 15:8759-8763. 10.7314/apjcp.2014.15.20.8759

4. Riedell PA, Smith SM: Double hit and double expressors in lymphoma: definition and treatment. Cancer. 2018, 124:4622-4632. 10.1002/cncr.31646

5. Lu TX, Fan L, Wang L, et al.: MYC or BCL2 copy number aberration is a strong predictor of outcome in patients with diffuse large B-cell lymphoma. Oncotarget. 2015, 6:18374-18388. 10.18632/oncotarget.4073

6. Ye Q, Xu-Monette ZY, Tzankov A, et al.: Prognostic impact of concurrent MYC and BCL6 rearrangements and expression in de novo diffuse large B-cell lymphoma. Oncotarget. 2016, 7:2401-2416. 10.18632/oncotarget.6262

7. Copie-Bergman C, Cuillière-Dartigues P, Baia M, et al.: MYC-IG rearrangements are negative predictors of survival in DLBCL patients treated with immunochemotherapy: a GELA/LYSA study. Blood. 2015, 126:24662474. 10.1182/blood-2015-05-647602

8. Nagib RM, Ibrahim EM, El-Ashwah S: Double-expressor and triple-expressor lymphomas: are these prognostically distinct groups of diffuse large B-cell lymphoma?. Egypt J Pathol. 2019, 39:239-243. 10.4103/EGJP.EGJP_29_19

9. Naseem M, Asif M, Khadim MT, Ud-Din H, Jamal S, Shoaib I: The frequency of double expresser in selected cases of high grade diffuse large B-cell lymphomas. Asian Pac J Cancer Prev. 2020, 21:1103-1107. 10.31557/APJCP.2020.21.4.1103

10. Mehta A, Verma A, Gupta G, Tripathi R, Sharma A: Double hit and double expresser diffuse large B cell lymphoma subtypes: discrete subtypes and major predictors of overall survival. Indian J Hematol Blood Transfus. 2020, 36:627-634. 10.1007/s12288-019-01248-w

11. Rosenthal A, Younes A: High grade B-cell lymphoma with rearrangements of MYC and BCL2 and/or BCL6: double hit and triple hit lymphomas and double expressing lymphoma. Blood Rev. 2017, 31:37-42. 10.1016/j.blre.2016.09.004

12. Johnson NA, Slack GW, Savage KJ, et al.: Concurrent expression of MYC and BCL2 in diffuse large B-cell lymphoma treated with rituximab plus cyclophosphamide, doxorubicin, vincristine, and prednisone. J Clin Oncol. 2012, 30:3452-3459. 10.1200/JCO.2011.41.0985

13. Green TM, Young KH, Visco C, et al.: Immunohistochemical double-hit score is a strong predictor of outcome in patients with diffuse large B-cell lymphoma treated with rituximab plus cyclophosphamide, doxorubicin, vincristine, and prednisone. J Clin Oncol. 2012, 30:3460-3467. 10.1200/JCO.2011.41.4342

14. Nowakowski GS, Czuczman MS: ABC, GCB, and double-hit diffuse large B-cell lymphoma: does subtype make a difference in therapy selection?. Am Soc Clin Oncol Educ Book. 2015, 35:449-457. 10.14694/EdBook_AM.2015.35.e449 